\title{
Best Relay Selection in Cooperative Spectrum Sharing Systems with Multiple Primary Users
}

\author{
F. R. V. Guimarães ${ }^{\dagger}$, D. B. da Costa ${ }^{\dagger}$, M. Benjillali*, T. A. Tsiftsis ${ }^{\ddagger}$, and G. K. Karagiannidis ${ }^{\S}$ \\ ${ }^{\dagger}$ Wireless Telecommunications Research Group (GTEL), Federal University of Ceará (UFC), CE, Brazil \\ ${ }^{*}$ Communications Systems Department, INPT, Rabat, Morocco \\ ${ }_{\ddagger}^{\ddagger}$ Department of Electrical Engineering, Technological Educational Institute of Lamia, Lamia, Greece \\ $\S$ Department of Electrical \& Computer Engineering, Aristotle University of Thessaloniki, Thessaloniki, Greece \\ Emails: rafaelvg@gtel.ufc.br, danielbcosta@ieee.org, benjillali@ieee.org, tsiftsis@teilam.gr, geokarag@auth.gr
}

\begin{abstract}
Cooperative spectrum sharing systems (CSSSs) have recently received considerable attention from the wireless community due to their performance gains and spectrum utilization improvement when compared to traditional communication systems. Owing to this fact, we investigate the outage performance of CSSSs in the presence of multiple primary users (PUs). The secondary user (SU) network is composed by one source node, $N$ decode-and-forward (DF) relays, and one destination. A best relay selection strategy is performed where the selected relay is that which maximizes the end-to-end signal-to-noise ratio (SNR) and, simultaneously, satisfies the interference constraint imposed by the $M$ PU receivers. The communication between the $\mathrm{SU}$ source and SU destination is carried out through the help of one out of $N$ DF relays and also via direct link. Afterwards, the SU destination selects the best path between the direct and the relaying path by using selection combining technique. A closedform expression for the outage probability (OP) is derived, and an asymptotic analysis is carried out which reveals that the diversity order of the considered system equals $N+1$, showing that it is not affected neither by the number of $P U$ receivers nor by the interference threshold. The presented analytical expressions are corroborated by means of Monte Carlo simulations and insightful discussions are provided.
\end{abstract}

\section{INTRODUCTION}

Along the last decade, the wireless communications community has witnessed a considerable upsurge in the demand of new wireless services and applications. The necessity for higher throughput/reliability and for an efficient spectrum allocation has boosted the interest of developing new techniques to deal with these requirements. To this end, two promising techniques, namely cooperative diversity [1] and cognitive radio [2], were proposed and since then an impressive number of studies has been carried out. Briefly speaking, the main idea behind cooperative diversity is to emulate a virtual antenna array by the distributed transmission and processing of the information so that spatial diversity can be achieved from the use of single-antenna devices. Cognitive radio, by its turn, provides an efficient use of the radio spectrum by allowing unlicensed users, called secondary users (SUs), to utilize a partition of the spectrum initially allocated to the licensed users, called primary users (PUs). This spectrum access may be granted provided that the PU communication is not affected, which is done by adjusting the transmission power of the SUs according to an interference threshold, namely interference

The work was supported by CNPq (Proc. 302106/2011-1) and FUNCAP. temperature. The concurrent use of the radio spectrum by the PUs and SUs is known as spectrum sharing [3].

Several works have investigated the combined use of cooperative diversity and spectrum sharing systems. In [4], considering one SU source, one SU relay, one SU destination, and one PU receiver, the outage analysis of cooperative spectrum sharing systems (CSSSs) subject to Nakagami- $m$ fading was studied. In [5], assuming multiple SU relays, the outage probability (OP) of CSSSs was evaluated, where a relay selection criterion considering the interference constraint on the PU, was implemented. The authors in [6] investigated the OP of CSSSs considering the presence of a direct link and a single relay, while a selection combining (SC) receiver was used at the SU destination to select the best path between the direct and the relaying link. This work was later extended in [7] for Nakagami- $m$ fading. In [8], the outage performance of CSSSs with best relay selection scheme was examined, in which the best relay was chosen based on the maximum instantaneous signal-to-noise ratio (SNR) from the $\mathrm{SU}$ relays to the $\mathrm{SU}$ destination (i.e., the channel state information from the SU source to the SU relays was not taken into consideration for the relay selection process).

In this paper, we investigate the outage performance of CSSSs composed of one SU source, multiple decode-andforward (DF) SU relays, one SU destination, and multiple PU receivers. It is assumed that a direct link between SU source and SU destination is available. Because of the spectrum sharing environment, a maximum transmission power at the SU nodes is imposed, which is defined by the maximum power available at the SU node and by the interference level at the PU receivers. In addition, a best relay selection strategy is performed where the selected relay will be the one maximizing the end-to-end SNR and, simultaneously, satisfying the interference threshold imposed by the $M \mathrm{PU}$ receivers. A closed-form expression for the outage probability (OP) is derived, and an asymptotic analysis is carried out which reveals that the diversity order of the considered system equals $N+1$, showing that it is not affected neither by the number of PU receivers nor by the interference threshold. The presented analytical expressions are corroborated by means of Monte Carlo simulations and insightful discussions are provided.

The remainder of this paper is organized as follows. In 


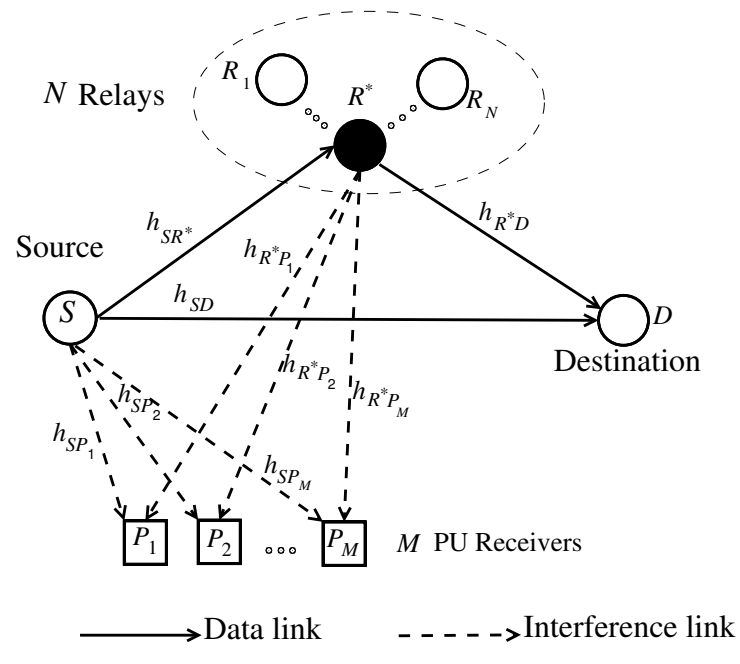

Fig. 1. Schematic of the system model.

Section II, the system and channel models are introduced along with the relay selection process. In Section III, a closed-form expression for the OP of the secondary network is derived. Also, an asymptotic analysis is carried out, in which the diversity gain is attained. In Section IV, representative numerical results are shown and validated by means of Monte Carlo simulations, and insightful discussions are drawn. Finally, in Section $\mathrm{V}$ the conclusions are drawn.

Throughout this paper, $f_{Z}(\cdot)$ and $F_{Z}(\cdot)$ denote the probability density function (PDF) and cumulative distribution function (CDF) of an arbitrary random variable (RV) $Z$, respectively, $h_{A B}$ stands for the channel coefficient between two arbitrary nodes $A$ and $B, \operatorname{Pr}(\cdot)$ indicates probability, and $E[\cdot]$ denotes statistical expectation.

\section{System AND Channel Models}

We consider a CSSS consisted of one SU source $S, N$ DF SU relays $R_{n}(n=1, \ldots, N)$, one SU destination $D$, and $M$ PU receivers $P_{m}(m=1, \ldots, M)$, as shown in Fig. 1. PU transmitters are not taken into account since the focus of this paper is on the SU communication ${ }^{1}$. All nodes are equipped with a single antenna and operate in a half-duplex mode. It is also assumed that the direct link between $S$ and $D$ is available. The channel coefficients experience Rayleigh block fading, i.e., they do not change during the transmission of a block of data, but change independently from one block to another. We also consider that all noise terms are additive white Gaussian noise (AWNG) with mean power $N_{0}$.

The end-to-end SNR acquired through an arbitrary relay $R_{n}$ can be expressed as $\min \left[\gamma_{S R_{n}}, \gamma_{R_{n} D}\right]$, where $\gamma_{S R_{n}}=$ $W_{S}\left|h_{S R_{n}}\right|^{2} / N_{0}$ and $\gamma_{R_{n} D}=W_{R_{n}}\left|h_{R_{n} D}\right|^{2} / N_{0}$ denote the instantaneous SNR of the links of the first-hop (i.e., from the SU source to the $n$-th SU relay) and the second-hop (i.e, from the $n$-th SU relay to the SU destination), respectively.

\footnotetext{
${ }^{1}$ As in previous works, we assume that the PU transmitters are located away from the SU nodes far enough not to impinge any interference upon the received signals at $R_{n}$ and $D$ and not to cause perturbation on the relay selection process.
}

Owing to the spectrum sharing environment, the transmit power constraints at the SU source and at the $n$-th SU relay can be written as [4], [5]

$$
\begin{aligned}
& W_{S} \leq \min \left(\min _{m} \frac{Q_{m}}{\left|h_{S P_{m}}\right|^{2}}, W\right), \\
& W_{R_{n}} \leq \min \left(\min _{m} \frac{Q_{m}}{\mid h_{\left.R_{n} P_{m}\right|^{2}}}, W\right),
\end{aligned}
$$

where $W$ is the maximum transmit power of the respective SU nodes and $Q_{m}$ designates the maximum tolerable interference power at the $m$-th PU receiver ${ }^{2}$.

Before the SU communication starts, one out of $N$ relays is selected to assist the communication process. To this end, we assume that global CSI including all channels is available at the SU source, and the best SU relay $R^{*}$ is chosen in such a way that the end-to-end SNR through the relaying link is maximized, i.e.,

$$
R^{*}=\arg \max _{n}\left[\min \left[\gamma_{S R_{n}}, \gamma_{R_{n} D}\right]\right]
$$

After the best SU relay is selected, the communication process starts and it comprises two phases. In phase I, the SU source broadcasts its information to both $R^{*}$ and $D$ with transmission power $W_{S}$. In phase II, assuming that the selected SU relay is always able to fully decode its received signal, $R^{*}$ forwards it to $D$ with a transmission power $W_{R_{n}}$. Concluded the twophase transmission, a SC strategy is performed by the SU destination. In this case, the path with the highest instantaneous SNR is selected between the direct and the best dual-hop link, so that the end-to-end SNR can be written as

$$
\gamma_{\mathrm{end}}=\max \left[\gamma_{S D}, \max _{n}\left[\min \left[\gamma_{S R_{n}}, \gamma_{R_{n} D}\right]\right]\right] \text {, }
$$

where $\gamma_{S D}=W_{S}\left|h_{S D}\right|^{2} / N_{0}$. Note that the two terms inside the operator $\max [\cdot, \cdot]$ in (3) are not statistically independent due to the presence of a common RV $\left|h_{S P_{m}}\right|^{2}$, which renders the analysis rather cumbersome. Following a similar analysis to [6] in order to deal with this inconvenience, next we derive the outage probability of the considered system in closed-form, from which an asymptotic analysis is carried out, followed by insightful discussions.

\section{Exact OUtage Probability And Asymptotic ANALYSIS}

As is well-known, the OP is defined as the probability that the end-to-end SNR, $\gamma_{\text {end }}$, falls bellow a predefined threshold, $\gamma_{\text {th }}$. As can be seen from (1) and (3), the terms $\gamma_{S D}$ and $\max _{n}\left[\min \left[\gamma_{S R_{n}}, \gamma_{R_{n} D}\right]\right]$ are not statistically independent because of the presence of a common term, $\left|h_{S P_{m}}\right|^{2}$. In what follows, for ease of presentation, we assume that $Q_{m}=Q$, $m=1, \ldots, M$, so that the expressions $\min _{m}\left(Q_{m} /\left|h_{S P_{m}}\right|^{2}\right)$ and $\min _{m}\left(Q_{m} /\left|h_{R_{n} P_{m}}\right|^{2}\right)$ are equivalent to $Q /\left(\max _{m}\left|h_{S P_{m}}\right|^{2}\right)$ and $Q /\left(\max _{m}\left|h_{R_{n} P_{m}}\right|^{2}\right)$, respectively.

\footnotetext{
${ }^{2}$ We assume that all PU receivers are located in an environment such that the signals from $S$ to $P_{m}$ experience independent identically distributed (i.i.d.) Rayleigh fading. The same can be said with respect to the signals from $R_{n}$ to $P_{m}$. However, the channels pertaining to the interference links $S$ $P_{m}$ experience Rayleigh fading conditions distinct from those of the links $R_{n}-P_{m}$.
} 
Let $X=\max _{m}\left|h_{S P_{m}}\right|^{2}$. Thus, relying on the conditional probability law, the conditional OP can be written as

$$
\begin{aligned}
& \operatorname{Pr}\left(\gamma_{\mathrm{end}}<\gamma_{\mathrm{th}} \mid X\right)=\overbrace{\operatorname{Pr}\left(\gamma_{S D}<\gamma_{\mathrm{th}} \mid X\right)}^{\varphi} \\
& \times \underbrace{\operatorname{Pr}\left(\max _{n}\left[\min \left[\gamma_{S R_{n}}, \gamma_{R_{n} D}\right]\right]<\gamma_{\mathrm{th}} \mid X\right)}_{\theta},
\end{aligned}
$$

As the channel gains are exponentially distributed, the first term of (4) can be calculated as

$$
\varphi=F_{\gamma_{S D}}\left(\gamma_{\mathrm{th}} \mid X\right)=1-e^{-\gamma_{\mathrm{th}} \lambda_{S D}},
$$

where $\lambda_{S D} \triangleq 1 / E\left[\gamma_{S D}\right]$. On the other hand, to determine $\theta$ in (4), note that, if $U$ and $V$ are two arbitrary RVs, then

$$
\operatorname{Pr}(\min [U, V]<\gamma)=F_{U}(\gamma)+F_{V}(\gamma)-F_{U}(\gamma) F_{V}(\gamma) .
$$

Thus, $\theta$ can be derived as

$$
\begin{aligned}
\theta= & \prod_{n=1}^{N}\left[\left(1-e^{-\gamma_{\mathrm{th}} \lambda_{S R_{n}}}\right)+\left(1-e^{-\gamma_{\mathrm{th}} \lambda_{R_{n} D}}\right)\right. \\
& \left.-\left(1-e^{-\gamma_{\mathrm{th}} \lambda_{S R_{n}}}\right)\left(1-e^{-\gamma_{\mathrm{th}} \lambda_{R_{n} D}}\right)\right],
\end{aligned}
$$

where $\lambda_{S R_{n}} \triangleq 1 / E\left[\gamma_{S R_{n}}\right]$ and $\lambda_{R_{n} D} \triangleq 1 / E\left[\gamma_{R_{n} D}\right]$. Let $Y=\max _{m}\left|h_{R_{n} P_{m}}\right|^{2}$. Using the total probability theorem, the OP can be determined as

$$
\begin{aligned}
P_{\text {out }}=\int_{0}^{\infty} \int_{0}^{\infty} & F_{\gamma_{S D}}\left(\gamma_{\mathrm{th}} \mid X\right) F_{\gamma_{S R^{*} D}}\left(\gamma_{\mathrm{th}} \mid X, Y\right) \\
& \times f_{X}(x) f_{Y}(y) d x d y
\end{aligned}
$$

where

$$
\gamma_{S R^{*} D}=\max _{n}\left[\min \left[\gamma_{S R_{n}}, \gamma_{R_{n} D}\right]\right]
$$

and

$$
\begin{aligned}
& f_{X}(x)=M\left(1-e^{-x \lambda_{S P}}\right)^{(M-1)} \lambda_{S P} e^{-x \lambda_{S P}}, \\
& f_{Y}(y)=M\left(1-e^{-y \lambda_{R_{n} P}}\right)^{(M-1)} \lambda_{R_{n} P} e^{-y \lambda_{R_{n} P}},
\end{aligned}
$$

with $\lambda_{S P} \triangleq 1 / E\left[\left|h_{S P_{m}}\right|^{2}\right]$ and $\lambda_{R_{n} P} \triangleq 1 / E\left[\left|h_{R_{n} P_{m}}\right|^{2}\right]$. Before solving (8), it is worth to see that

$$
\begin{aligned}
\min \left(\frac{Q}{X}, W_{S}\right) & = \begin{cases}W_{S}, & \text { when } X \leq Q / W_{S}, \\
Q / X, & \text { when } X>Q / W_{S},\end{cases} \\
\min \left(\frac{Q}{Y}, W_{R_{n}}\right) & = \begin{cases}W_{R_{n}}, & \text { when } Y \leq Q / W_{R_{n}}, \\
Q / Y, & \text { when } Y>Q / W_{R_{n}} .\end{cases}
\end{aligned}
$$

Based on (11) and (12), and in order to satisfy all the combinations of transmit power conditions, the OP can be calculated by expanding (8) into the sum of four terms, with each one being expressed as a double-integral. More specifically, $P_{\text {out }}$ can be rewritten as $P_{\text {out }}=\xi_{1}+\xi_{2}+\xi_{3}+\xi_{4}$, where

$$
\begin{aligned}
& \xi_{1}=\int_{0}^{Q / W} \int_{0}^{Q / W} F_{\gamma_{S D}}\left(\gamma_{\mathrm{th}} \mid X\right) F_{\gamma_{S R^{*} D}}\left(\gamma_{\mathrm{th}} \mid X, Y\right) \\
& \times f_{X}(x) f_{Y}(y) d x d y, \\
& \xi_{2}=\int_{0}^{Q / W} \int_{Q / W}^{\infty} F_{\gamma_{S D}}\left(\gamma_{\mathrm{th}} \mid X\right) F_{\gamma_{S R^{*} D}}\left(\gamma_{\mathrm{th}} \mid X, Y\right) \\
& \times f_{X}(x) f_{Y}(y) d x d y,
\end{aligned}
$$

$$
\begin{aligned}
& \xi_{3}=\int_{Q / W}^{\infty} \int_{0}^{Q / W} F_{\gamma_{S D}}\left(\gamma_{\mathrm{th}} \mid X\right) F_{\gamma_{S R^{*} D}}\left(\gamma_{\mathrm{th}} \mid X, Y\right) \\
& \times f_{X}(x) f_{Y}(y) d x d y, \\
& \xi_{4}=\int_{Q / W}^{\infty} \int_{Q / W}^{\infty} F_{\gamma_{S D}}\left(\gamma_{\mathrm{th}} \mid X\right) F_{\gamma_{S R^{*} D}}\left(\gamma_{\mathrm{th}} \mid X, Y\right) \\
& \times f_{X}(x) f_{Y}(y) d x d y,
\end{aligned}
$$

where $W_{S}=W_{R_{n}}=W$ is assumed. By making the appropriate substitutions in (13) and using the binomial expansion for re-expressing the PDFs of $X$ and $Y$ given in (10), we have

$$
\begin{gathered}
\xi_{1}=\left(1-e^{-\gamma_{\mathrm{th}} \lambda_{S D}^{W}}\right) \prod_{n=1}^{N}\left(1-e^{-\gamma_{\mathrm{th}}\left(\lambda_{S R_{n}}^{W}+\lambda_{R_{n} D}^{W}\right)}\right) \\
\times M^{2} \sum_{m=0}^{M-1} \sum_{t=0}^{M-1}(-1)^{m}(-1)^{t}\left(\begin{array}{c}
M-1 \\
m
\end{array}\right)\left(\begin{array}{c}
M-1 \\
t
\end{array}\right) \\
\times \frac{\left(1-e^{\left(-\frac{Q}{W} \lambda_{S P}(m+1)\right)}\right)}{\lambda_{S P}(m+1)} \frac{\left(1-e^{\left(-\frac{Q}{W} \lambda_{R_{n} P}(t+1)\right)}\right)}{\lambda_{R_{n} P}(t+1)} .
\end{gathered}
$$

where

$$
\lambda_{I J}^{W} \triangleq \frac{1}{E\left[W\left|h_{I J}\right|^{2} / N_{0}\right]},
$$

with $I \in\left\{S, R_{n}\right\}$ and $J \in\left\{R_{n}, D\right\}$. In the same way, by making the appropriate substitutions in (14), it follows that

$$
\begin{aligned}
\xi_{2} & =\int_{0}^{Q / W} \int_{Q / W}^{\infty}\left(1-e^{-\gamma_{\mathrm{th}} \lambda_{S D}^{W}}\right) \prod_{n=1}^{N}\left(1-e^{-\gamma_{\mathrm{th}}\left(\lambda_{S R_{n}}^{W}+y \lambda_{R_{n} D}^{Q}\right)}\right) \\
& \times M\left(1-e^{-x \lambda_{S P}}\right)^{(M-1)} \lambda_{S P} e^{-x \lambda_{S P}} M\left(1-e^{-y \lambda_{R_{n} P}}\right)^{(M-1)} \\
& \times \lambda_{R_{n} P} e^{-y \lambda_{R_{n} P}} d x d y
\end{aligned}
$$

where $y \lambda_{R_{n} D}^{Q} \triangleq 1 / E\left[(Q / y)\left(\left|h_{R_{n} D}\right|^{2} / N_{0}\right)\right]$. To proceed further, we rely on the following identity

$$
\prod_{k=1}^{K}\left(1-x_{k}\right)=\sum_{k=0}^{K} \frac{(-1)^{k}}{k !} \sum_{\substack{n_{1}=\ldots=n_{k} \\ n_{1}<\ldots<n_{k}}}^{K} \ldots \prod_{t=1}^{K} x_{n_{t}} .
$$

Since the SU relays are assumed to be clustered relatively close to each other [9], this allows us to say that all links from $S$ to $R_{n}$ have the same average SNR, (i.e., $\lambda_{S R_{n}}=\lambda_{S R}, \forall n$ ). The same can be assumed for the links from $R_{n}$ to $D$. Thus, by plugging (20) into (19), $\xi_{2}$ can be derived as (21), given at the top of the next page. Finally, in order to avoid repetition of the analytical derivation steps, (15) and (16) can also be obtained in closed-form as (22) and (23), respectively, where $\lambda_{S R_{n}}^{Q} \triangleq$ $1 / E\left[Q\left|h_{S R_{n}}\right|^{2} / N_{0}\right]$ and $\lambda_{S D}^{Q} \triangleq 1 / E\left[Q\left|h_{S D}\right|^{2} / N_{0}\right]$.

Finally, by substituting (17), (21), (22), and (23) into $P_{\text {out }}$, a closed-form expression for the outage probability is derived. To the best of the authors' knowledge, this expression has not been reported in the literature yet.

In order to gain further insights from the derived expressions, an asymptotic analysis (high-SNR regime) will be presented, from which the diversity gain of the considered system will be obtained. It will be shown, for example, that the diversity gain is not affected neither by the number of PU receivers nor by the interference threshold. 


$$
\begin{aligned}
\xi_{2} & =M^{2} \sum_{m=0}^{M-1} \sum_{t=0}^{M-1} \sum_{n=0}^{N}(-1)^{(m+t+n)}\left(\begin{array}{c}
M-1 \\
m
\end{array}\right)\left(\begin{array}{c}
M-1 \\
t
\end{array}\right)\left(\begin{array}{c}
N \\
n
\end{array}\right) \lambda_{R_{n} P}\left(1-e^{-\gamma_{\mathrm{th}} \lambda_{S D}^{W}}\right) \frac{\left(1-e^{\left(-\frac{Q}{W} \lambda_{S P}(m+1)\right)}\right)}{\lambda_{S P}(m+1)} \\
& \times \frac{\exp \left(-\gamma_{\mathrm{th}} n\left(\lambda_{S R_{n}}^{W}+\frac{Q}{W} \lambda_{R_{n} D}^{Q}\right)-\frac{Q}{W} \lambda_{R_{n} P}(t+1)\right)}{\gamma_{\mathrm{th}} n \lambda_{R_{n} D}^{Q}+\lambda_{R_{n} P}(t+1)} .
\end{aligned}
$$

$$
\begin{aligned}
\xi_{3} & =\sum_{l=0}^{1} \sum_{m=0}^{M-1} \sum_{t=0}^{M-1} \sum_{n=0}^{N} M^{2}(-1)^{(m+t+n+l)}\left(\begin{array}{c}
M-1 \\
m
\end{array}\right)\left(\begin{array}{c}
M-1 \\
t
\end{array}\right)\left(\begin{array}{c}
N \\
n
\end{array}\right) \lambda_{R_{n} P} \frac{\left(1-e^{\left(-\frac{Q}{W} \lambda_{R_{n} P}(t+1)\right)}\right)}{\lambda_{R_{n} P}(t+1)} \exp \left(-l \frac{Q}{W} \gamma_{\mathrm{th}} \lambda_{S D}^{Q}\right) \\
& \times \lambda_{S P} \frac{\exp \left(-n \gamma_{\mathrm{th}}\left(\frac{Q}{W} \lambda_{S R_{n}}^{Q}+\lambda_{R_{n} D}^{W}\right)-\frac{Q}{W} \lambda_{S P}(m+1)\right)}{l \gamma_{\mathrm{th}} \lambda_{S D}^{Q}+n \gamma_{\mathrm{th}} \lambda_{S R_{n}}^{Q}+\lambda_{S P}(m+1)} .
\end{aligned}
$$

$$
\begin{aligned}
\xi_{4} & =\sum_{l=0}^{1} \sum_{m=0}^{M-1} \sum_{t=0}^{M-1} \sum_{n=0}^{N} M^{2}(-1)^{(m+t+n+l)}\left(\begin{array}{c}
M-1 \\
m
\end{array}\right)\left(\begin{array}{c}
M-1 \\
t
\end{array}\right)\left(\begin{array}{c}
N \\
n
\end{array}\right) \lambda_{R_{n} P} \exp \left(-l \frac{Q}{W} \gamma_{\mathrm{th}} \lambda_{S D}^{Q}\right) \exp \left(-\frac{Q}{W} \lambda_{R_{n} P}(t+1)\right) \\
& \times \frac{1}{n \gamma_{\mathrm{th}} \lambda_{R_{n} D}^{Q}+\lambda_{R_{n} P}(t+1)} \frac{\exp \left(-n \gamma_{\mathrm{th}} \frac{Q}{W}\left(\lambda_{S R_{n}}^{Q}+\lambda_{R_{n} D}^{Q}\right)-\frac{Q}{W} \lambda_{S P}(m+1)\right)}{l \gamma_{\mathrm{th}} \lambda_{S D}^{Q}+n \gamma_{\mathrm{th}} \lambda_{S R_{n}}^{Q}+\lambda_{S P}(m+1)} .
\end{aligned}
$$

\section{A. Asymptotic Analysis}

Let $\bar{\gamma} \triangleq 1 / N_{0}$ be the system SNR and assume that $Q / W=$ $\mu$, where $\mu$ is a positive constant. By using the MacLaurin series of exponential functions and noting that, when $\bar{\gamma} \rightarrow \infty$, $\lambda_{S P} \gg \frac{\gamma_{\mathrm{th}}}{\bar{\gamma}}$ and $\lambda_{R_{n} P} \gg \frac{\gamma_{\mathrm{th}}}{\bar{\gamma}}$, an asymptotic expression for each term $\xi_{i}$ can be derived as

$$
\begin{aligned}
P_{\mathrm{out}}^{\xi_{1}} \simeq & \left(\gamma_{\mathrm{th}} \lambda_{S D}^{W}\right) M^{2} \sum_{m=0}^{M-1} \sum_{t=0}^{M-1}(-1)^{m+t}\left(\begin{array}{c}
M-1 \\
m
\end{array}\right)\left(\begin{array}{c}
M-1 \\
t
\end{array}\right) \\
& \times \frac{\left(1-e^{\left(-\mu \lambda_{S P}(m+1)\right)}\right)}{\lambda_{S P}(m+1)} \frac{\left(1-e^{\left(-\mu \lambda_{R_{n}}(t+1)\right)}\right)}{\lambda_{R_{n} P}(t+1)} \\
& \times \prod_{n=1}^{N}\left[\gamma_{\mathrm{th}}\left(\lambda_{S R_{n}}^{W}+\lambda_{R_{n} D}^{W}\right)\right] \propto\left(\frac{1}{\bar{\gamma}}\right)^{N+1}, \\
P_{\mathrm{out}}^{\xi_{2}} \simeq & \left(\gamma_{\mathrm{th}} \lambda_{S D}^{W}\right) M^{2} \sum_{m=0}^{M-1} \sum_{t=0}^{M-1}(-1)^{m+t}\left(\begin{array}{c}
M-1 \\
m
\end{array}\right)\left(\begin{array}{c}
M-1 \\
t
\end{array}\right) \\
& \times \frac{\left(1-e^{\left(-\mu \lambda_{S P}(m+1)\right)}\right)}{\lambda_{S P}(m+1)} \frac{e^{\left(-\mu \lambda_{R_{n} P}(t+1)\right)}}{\lambda_{R_{n} P}(t+1)} \\
& \times \prod_{n=1}^{N}\left[\gamma_{\mathrm{th}}\left(\lambda_{S R_{n}}^{W}+\mu \lambda_{R_{n} D}^{Q}\right)\right] \propto\left(\frac{1}{\bar{\gamma}}\right)^{N+1}, \\
P_{\mathrm{out}}^{\xi_{3}} \simeq & \left(\mu \gamma_{\mathrm{th}} \lambda_{S D}^{Q}\right) M^{2} \sum_{m=0}^{M-1} \sum_{t=0}^{M-1}(-1)^{m+t}\left(\begin{array}{c}
M-1 \\
m
\end{array}\right)\left(\begin{array}{c}
M-1 \\
t
\end{array}\right) \\
& \times \frac{\left(1-e^{\left(-\mu \lambda_{R_{n} P}(t+1)\right)}\right)}{\lambda_{R_{n} P(t+1)} \frac{e^{\left(-\mu \lambda_{S P}(m+1)\right)}}{\lambda_{S P}(m+1)}} \\
& \times \prod_{n=1}^{N}\left[\gamma_{\mathrm{th}}\left(\mu \lambda_{S R_{n}}^{Q}+\lambda_{R_{n} D_{l}}^{W}\right)\right] \propto\left(\frac{1}{\bar{\gamma}}\right)^{N+1},
\end{aligned}
$$

$$
\begin{aligned}
P_{\mathrm{out}}^{\xi_{4}} \simeq & \left(\mu \gamma_{\mathrm{th}} \lambda_{S D}^{Q}\right) M^{2} \sum_{m=0}^{M-1} \sum_{t=0}^{M-1}(-1)^{m+t}\left(\begin{array}{c}
M-1 \\
m
\end{array}\right)\left(\begin{array}{c}
M-1 \\
t
\end{array}\right) \\
& \times \frac{\left(e^{\left(-\mu \lambda_{R_{n} P}(t+1)\right)}\right)}{\lambda_{R_{n} P}(t+1)} \frac{e^{\left(-\mu \lambda_{S P}(m+1)\right)}}{\lambda_{S P}(m+1)} \\
& \times \prod_{n=1}^{N}\left[\mu \gamma_{\mathrm{th}}\left(\lambda_{S R_{n}}^{Q}+\lambda_{R_{n} D_{l}}^{Q}\right)\right] \propto\left(\frac{1}{\bar{\gamma}}\right)^{N+1}
\end{aligned}
$$

From the analytical expressions above, it is easy to see that the system achieves full diversity order, which is $N+1$. It is worth noting that the interference constraints have no influence on the diversity gain.

\section{Numerical RESUlts AND Discussions}

In this Section, some representative numerical examples are shown in order to validate the proposed analysis. As in previous works, we consider a two-dimensional plane for the location of all nodes pertaining to the primary and secondary networks, in which without any loss of generality the SU source is placed at $(0,0)$, the $N$ SU relays are clustered together and collocated at $(0.5,0)$, the $\mathrm{SU}$ destination is located at $(1,0)$, and the $M$ PU receivers are also clustered together and placed at $(0,1)$. The statistical average of the channel gains between any two nodes is determined by $d^{-\rho}$, with $d$ denoting the distance between the respective nodes and $\rho$ being the path loss coefficient, which is set to $\rho=4$. Also, the outage threshold, $\gamma_{\text {th }}$, is set to $3 \mathrm{~dB}$. As will be observed, all the investigated cases reveal an excellent agreement between analytical and simulation results.

Fig. 2 shows the exact and asymptotic outage behaviors of the CSSS under study assuming $N=3$ relays and different numbers of PU receivers. As expected, when the number of PU receivers increases, the outage performance decreases. 


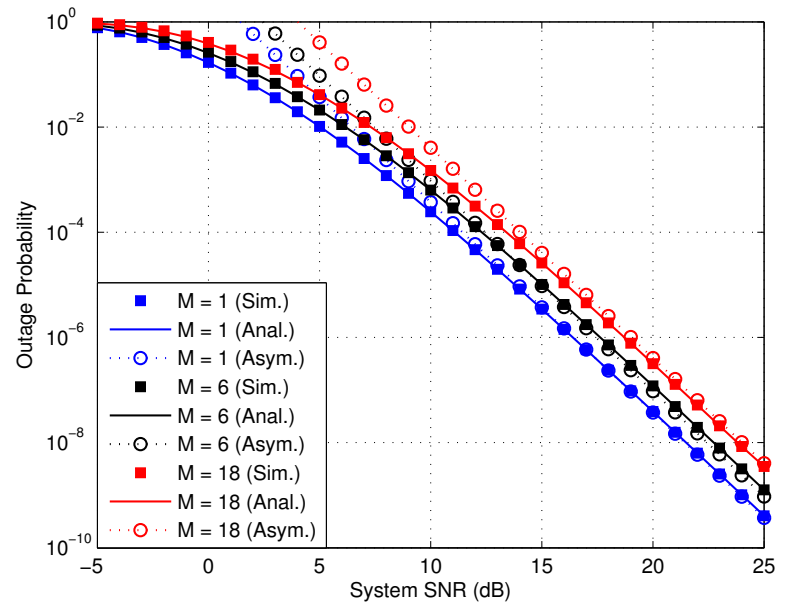

Fig. 2. Outage probability and asymptotic behavior for different number of PU receivers for $N=3$ relays $(Q=W=0.5)$.

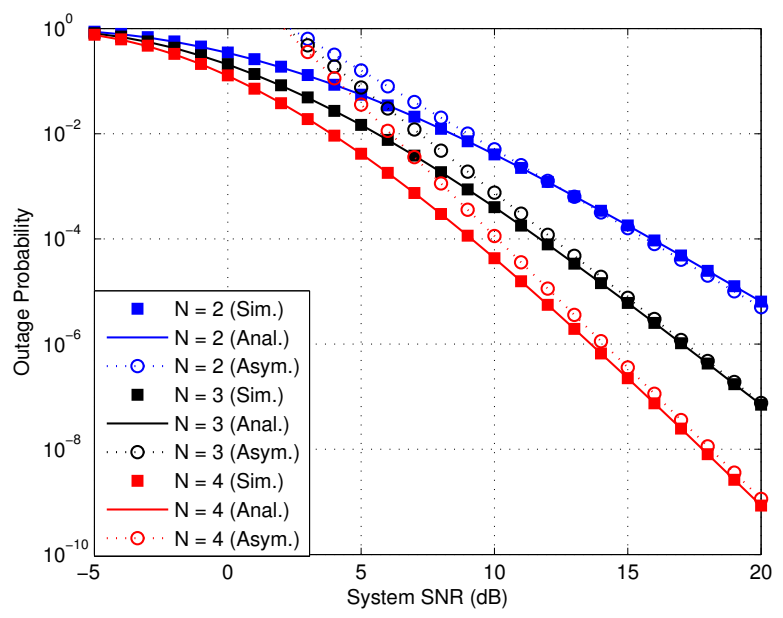

Fig. 3. Outage probability and asymptotic behavior for different number of SU relays for $M=3$ PU receivers $(Q=W=0.5)$.

However, it can be seen that the number of PU receivers has no influence on the diversity order of the system, which is equal to $N+1$.

Differently from Fig. 2, in Fig. 3 we fix the number of PU receivers, $M=3$, and vary the number of SU relays. As expected, by increasing $N$, the outage performance as well as the diversity order are improved, showing that the cooperative diversity has a great benefit for the system performance. In these two figures, note that the asymptotic curves are very tight with the exact curves at high-SNR regions, which confirms the correctness of our analysis.

Fig. 4 shows how the temperature constraint, $Q$, influences the outage performance. By assuming $M=N=3$, we see that the system becomes saturated after a certain value of $W / N_{0}$. This happens because the system reaches the maximum allowed transmission power. Also, as the temperature constraint gets higher, the system behavior approaches the "non-interference" case.

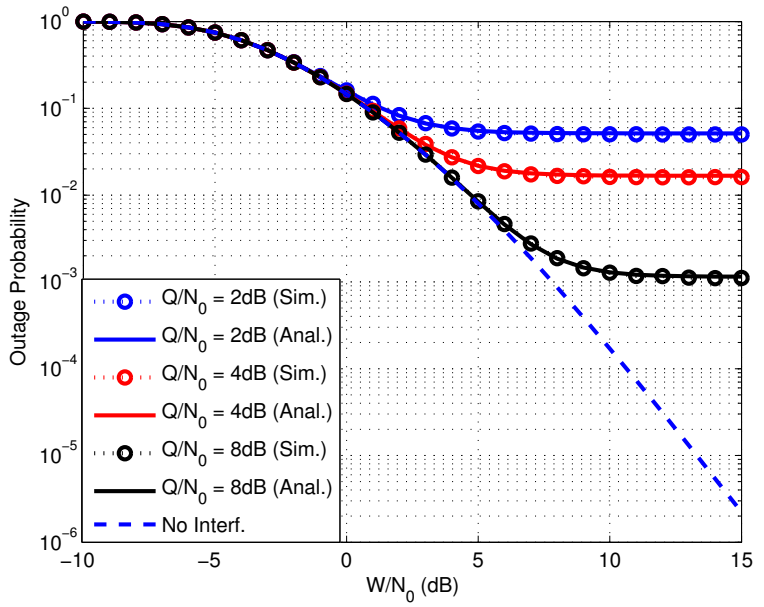

Fig. 4. Impact of interference constraints on the $\mathrm{OP}(M=N=3)$.

\section{Conclusions}

The outage performance of CSSSs in the presence of direct link was investigated. Employing a best relay selection strategy, a closed-form expression for the OP was derived, from which an asymptotic analysis was carried out and revealed that the considered system achieves full diversity order. It was observed that: (i) neither the number of PU receivers nor the temperature interference have influence on the diversity order of the system, and (ii) the interference constraint imposed by the PU receivers causes a saturation phenomenon at the outage probability. To the best of the authors' knowledge, this kind of analysis has not been performed in the literature yet, which renders it very important for the system design of future cognitive relay networks subject to multiple primary constraints.

\section{REFERENCES}

[1] J. N. Laneman, D. N. C. Tse and G. W. Wornell, "Cooperative diversity in wireless networks: Efficient protocols and outage behavior," IEEE Trans. Inf. Theory, vol. 50, no. 12, pp. 3062- 3080, Dec. 2004.

[2] J. Mitola et al., "Cognitive radio: making software radios more personal," IEEE Pers. Commun, vol. 6, no. 4, pp. 13-18, 1999.

[3] A. Ghasemi and E. S. Sousa, "Fundamental limits of spectrum-sharing in fading environments," IEEE Trans. Wireless Commun., vol. 6, no. 2, pp. 649-658, Feb. 2007.

[4] C. Zhong, T. Ratnarajah, and K.-K. Wong, "Outage analysis of decodeand-forward cognitive dual-hop systems with the interference constraint in Nakagami-m fading channels," IEEE Trans. Veh. Technol., vol. 60, no. 6, pp. 2875-2879, Jul. 2011.

[5] J. Lee, H. Wang, J. G. Andrews and D. Hong, "Outage probability of cognitive relay networks with interference constraints," IEEE Trans. Wireless Commun., vol. 10, no. 2, pp. 390-395, Feb. 2011.

[6] T. Q. Duong, V. N. Q. Bao, G. C. Alexandropoulos, and H. -J. Zepernick, "Cooperative spectrum sharing networks with AF relay and selection diversity," IET Electron. Lett., vol. 47, no. 20, Sep. 2011.

[7] T. Q. Doung, D. B. da Costa, M. Elkashlan and V. N. Q. Bao, "Cognitive amplify-and-forward relay networks over Nakagami- $m$ fading," IEEE Trans. Veh. Technol., vol. 61, no. 5, pp. 2368-2374, Jun. 2012.

[8] L. Luo, P. Zhang, G. Zhang, and J. Qin, "Outage performance for cognitive relay networks with underlay spectrum sharing," IEEE Commun. Lett., vol. 15, no. 7, pp. 710-712, Jul. 2011.

[9] D. B. da Costa and S. Aissa, "End-to-end performance of dual-hop semiblind relaying systems with partial relay selection," IEEE Trans. Wireless Commun., vol. 8, no. 8, pp. 4306-4315, Aug. 2009. 